\title{
Cancer/testis antigens (CTAs) expression in resected lung cancer
}

This article was published in the following Dove Press journal:

OncoTargets and Therapy

\author{
Shi Jin',* \\ Shoubo Cao ${ }^{1,2, *}$ \\ Jianhua $\mathrm{Li}^{3}$ \\ Qingwei Meng' \\ Chunyan Wang ${ }^{1,2}$ \\ Lei Yao ${ }^{4}$ \\ Yaoguo Lang ${ }^{4}$ \\ Jingyan $\mathrm{Cao}^{\prime}$ \\ Jing Shen' \\ Bo Pan \\ Jing $\mathrm{Hu}^{\prime}$ \\ Yan Yu' \\ 'Department of Medical Oncology, \\ Harbin Medical University Cancer \\ Hospital, Harbin, China; ${ }^{2}$ Department \\ of Medical Oncology, Linyi People's \\ Hospital, Linyi, China; ${ }^{3}$ Department \\ of Neurosurgery, The Fourth \\ Affiliated Hospital of Harbin \\ Medical University, Harbin, China; \\ ${ }^{4}$ Department of Thoracic Surgery, \\ Harbin Medical University Cancer \\ Hospital, Harbin, China \\ *These authors contributed equally \\ to this work
}

\begin{abstract}
Background: Increasing evidence shows cancer/testis antigens (CTAs) play a key role in oncogenesis. Our pre-study finds that MAGEA1, MAGEA10, MAGEB2, KK-LC-1, and CTAG1A/B have high expression frequencies at the protein level. We aim to explore their prognostic role and correlations with clinical characteristics in resected lung cancer at the mRNA level.

Methods: Thirty-eight surgical lung cancer samples were included. Validation study was performed based on The Cancer Genome Atlas database. The prognostic roles of CTAs were evaluated by Kaplan-Meier and multivariate analysis.

Results: High expression of MAGEA1 (16.7\% vs 65.0\%, $P=0.004)$, MAGEA10 (61.1\% vs 95.0\%, $P=0.016), M A G E B 2$ (55.6\% vs $95.0 \%, P=0.007)$, and $K K-L C-1$ ( $16.7 \%$ vs $55.0 \%, P=0.020)$ was closely correlated with lymph node metastasis at diagnosis. Patients with TNM stage II or III had a higher expression of MAGEA10 (57.1\% vs $91.7 \%, P=0.034)$ and $K K-L C-1(14.3 \%$ vs $50.0 \%, P=0.039$ ) compared with patients in TNM stage I. High $C T A G 1 A / B$ expression showed unfavorable prognosis in all cases $(P<0.05)$. Subgroup analysis showed high $C T A G 1 A / B$ expression was a negative prognostic factor of survival $(P=0.031)$ in patients with TNM stage II or III. Although no statistical significance was reached, high $C T A G 1 A / B$ also showed a similar prognostic trend in lung adenocarcinoma (ADC) and squamous cell carcinoma. The Cancer Genome Atlas database showed the negative prognostic role of $C T A G 1 A / B$ was mainly induced by CTAG1B (NY-ESO-1, $P=0.047$ ) and high CTAG1B expression (hazard ratio $=2.733,95 \% \mathrm{CI}$ : 1.348-5.541, $P=0.005)$ was an independent negative prognostic factor of lung ADC.

Conclusion: CTAs represent potential candidate targets for immunotherapy and their expression was closely correlated with tumor stage. High CTAG1B expression was an independent negative prognostic factor of lung ADC.
\end{abstract}

Keywords: lung cancer, cancer/testis antigens, immunotherapy, prognosis

\section{Introduction}

With the aging and growth of population as well as the prevalence of established risk factors, cancer incidence and mortality have been increasing. ${ }^{1}$ GLOBOCAN 2012 data shows lung cancer remains the first leading cause of cancer-related death in male patients worldwide. In female patients of more developed countries, it has even surpassed breast cancer to be the leading cause of cancer death. ${ }^{1}$ There is no doubt that cancer will create a giant burden on societies in the following decades.

In a recent study, it is found that adjuvant gefitinib can significantly prolong the disease-free survival (28.7 vs 18.0 months, $P=0.0054$ ) of resected stage II-III $E G F R$-mutant non-small-cell lung cancer compared with traditional chemotherapy. Its impact on overall survival (OS) need to be further studied because the OS data are not yet mature. ${ }^{2}$ Studies involving Nivolumab ( $P D-1$ inhibitor) have achieved great success and the results show that Nivolumab can significantly improve the OS of
Correspondence: Yan Yu

Department of Medical Oncology, Harbin Medical University Cancer Hospital, I50 Haping Road, Harbin I5008I, China Tel +8645186298727

Fax +8645186298727

Email yuyan@ems.hrbmu.edu.cn (c)
hereby accept the Terms. Non-commercial uses of the work are permitted without any further permission from Dove Medical Press Limited, provided the work is properly attributed. For permission for commercial use of this work, please see paragraphs 4.2 and 5 of our Terms (https://www.dovepress.com/terms.php).
forition 
lung cancer patients with a favorable safety profile. ${ }^{3-5}$ Lung cancer treatment has become more and more standardized and patients may have more chances of being treated with variable treatment strategies. However, the prognosis of lung cancer remains unsatisfactory, with a 5-year survival rate of $<10 \%{ }^{6}$ Treatment strategies with high efficiency and low toxicity are urgently needed.

Immunotherapy has been an active area in cancer research in recent years. However, the identification of ideal targets is the main point restricting the study of immunotherapy. Cancer/testis antigens (CTAs) are considered ideal targets for cancer treatment due to their high immunogenic, restricted expression in germ cells and malignancies. ${ }^{7-9}$ Generally, germ cells do not express human leukocyte antigen (HLA) class I molecules, leading them escape from the attack of T cells. Though somatic tissues, such as thyroid, breast, and retina may have the expression of CTAs, their expression is far below that in testis tissue and their function remains to be discovered. CTA-induced immunotherapy can specifically identify and kill cancer cells expressing corresponding CTAs, while they have limited toxicities on patients at the same time. ${ }^{10-12}$

One important point needing to be considered when selecting ideal targets for immunotherapy is the expression frequency of CTAs. Our previous study (unpublished data) shows that MAGEA1, MAGEA10, MAGEB2, KK-LC-1, and $C T A G 1 A / B$ have a high expression rate in lung cancer samples. Increasing evidence has demonstrated that CTAs play important roles in oncogenesis, including sustained growth, angiogenesis, evading apoptosis, invasion, metastasis, and so on. MAGEA, MAGEB, and MAGEC families have been demonstrated to promote cancer cells survival and inhibit apoptosis through the binding and regulation of tumor suppressor p53. ${ }^{13}$ CTAs, including CTAG1B (NY-ESO-1), $S S X$, and so on also play a key role in tumor invasion and metastasis. ${ }^{14} M A G E A 1$ and $C T A G 1 B$ show higher expression frequencies in metastatic melanoma compared with primary tumor. ${ }^{15,16} K K-L C-1$ is a novel CTA that was first identified in human lung cancer, and is also expressed in a wide range of malignancies. ${ }^{17}$ CTAs expression is heterogeneous and their expression has significant regional and racial distinctions. ${ }^{9,18-23}$ In this study, we aim to analyze the expression rate of the aforementioned 5 CTAs and explore their relationship with clinical features in resected lung cancer patients of China.

\section{Patients and methods}

\section{Patients and specimens}

This study used 38 lung cancer samples for real-time polymerase chain reaction (RT-PCR). All patients had pathologically confirmed lung cancer and the tissues were surgically obtained at our hospital between May 2013 and August 2013. Our study was approved by the Ethics Committee of Harbin Medical University Cancer Hospital and written informed consent was obtained from each patient. The tumor stage was classified based on the eighth edition of lung cancer stage classification. ${ }^{24}$

\section{Quantitative real time-PCR}

Five relative high expressed CTAs (MAGEA1, MAGEA10, $M A G E B 2, K K-L C-1$, and $C T A G 1 A / B)$ in our pre-study were selected to examine their expression at mRNA level. Total RNA from lung cancer frozen tissue specimens was extracted using TRIZOL reagent (Invitrogen, Carlsbad, CA, USA) according to the manufacturer's protocol. Complementary DNA was synthesized using the High Capacity cDNA Reverse Transcription Kit (Applied Biosystems, Foster City, CA, USA) in a final reaction volume of $20 \mu \mathrm{L}$. RT-PCR was performed using SYBR Green Master Mix (Roche Applied Science, Mannheim, Germany) and QuantStudio ${ }^{\circledR}$ 3 Thermo Fisher (Applied Biosystems). $\beta$-actin was used as the internal control. The primers were synthesized by Oligofectamine (Invitrogen, San Diego, CA, USA). There were no nonspecific amplification products observed in any of the reactions based on the analysis of the dissociation curves. Each RT-PCR experiment was repeated 3 times. Ct represents the threshold cycle. The data were normalized to the $\beta$-actin expression levels, which was named $\triangle \mathrm{Ct}$ value. The sample with the largest $M A G E A 1, M A G E A 10, M A G E B 2, K K-L C-1$, and $C T A G 1 A / B \triangle \mathrm{Ct}$ was used as control and the relative gene expression levels were calculated using the comparative $\mathrm{Ct}(\Delta \Delta \mathrm{Ct})$ method. The relative expression was calculated as $2^{-\Delta \Delta \mathrm{Ct}}$. High or low CTAs expression was determined by a receiver operating characteristic (ROC) curve analysis according to the lymph node metastasis status at diagnosis (patients with lymph node metastasis at diagnosis were registered as 1 , otherwise 0 ). Youden index was calculated as (sensitivity + specificity -1 ). The optimal cut-off value was determined as CTA expression with the largest Youden index. High expression was determined as CTA expression larger than the optimal cut-off value, and low expression as CTA expression lower than the optimal cut-off value. The primer sets are seen in Table 1.

\section{The Cancer Genome Atlas (TCGA) data}

RNA-seq data of these CTAs for lung cancer and corresponding clinical information were downloaded from the TCGA database. Patients with missing clinicopathological data, including age, gender, tumor stage, OS, and survival status 
Table I Polymerase chain reaction amplification programs

\begin{tabular}{lll}
\hline Gene & Direction & Primers from $\mathbf{5}^{\prime}$ to $\mathbf{3}^{\prime}$ \\
\hline MAGEAI & Forward & ATCAACTTCACTCGACAGAGGC \\
& Reverse & CGGAACAAGGACTCCAGGATAC \\
MAGEAIO & Forward & GTCCAAGCACCCTACAGGTC \\
& Reverse & TTCTGCCTTTGTGATCGGCT \\
MAGEB2 & Forward & GAGCCAGAGTTGTAGCCAGG \\
& Reverse & AGACCTCATTGGGGGTGAGA \\
KKLCI & Forward & ACTGCTTCCCAACTACCAGC \\
& Reverse & ACACAGAATGCTGCTCGCTA \\
CTAGIA/B & Forward & TGTCCGGCAACATACTGACT \\
& Reverse & ACTGCGTGATCCACATCAAC \\
$\beta$-actin & Forward & CAGAAGGATTCCTATGTGG \\
& Reverse & CATGATCTGGGTCATCTTC \\
\hline
\end{tabular}

were excluded. High and low CTAs (MAGEA1, MAGEA10, $M A G E B 2$, and $K K-L C-1)$ expression was also determined by ROC curve analysis used previously. However, in total cases, only few patients in TCGA database expressed CTAG1A $(\mathrm{n}=106)$ and CTAG1B $(\mathrm{n}=43)$, and the relative expression levels were low, especially in lung squamous cell carcinoma (SCC) patients. Patients with CTAG1A and CTAG1B relative expression levels $>0$ were registered as high expression, whilst those with levels $\leq 0$ were registered as low expression.

\section{Statistical analysis}

The OS time was defined from diagnosis to death or the last follow-up if the patients were still alive. The survival curves and 95\% CIs were obtained using the Kaplan-Meier method, and the survival in different groups was compared by log-rank tests. The relationships between CTAs and clinicopathological features were assessed by the chi-squared test. The univariate analysis was used to evaluate the potential prognostic role of variables. The prognostic factors with $P$-values $<0.05$ in the univariate analysis were examined in the multivariate analysis. SPSS 16.0 statistical software was used for statistical analysis. All $P$-values $<0.05$ were considered statistically significant.

\section{Results}

\section{Patient characteristics}

The patient characteristics are listed in Table 2. The mean age for all cases was $56.53 \pm 7.76$ years (range $32-71$ ). Most patients were male $(68.4 \%, \mathrm{n}=26)$ with an age of $<60$ years at diagnosis $(63.2 \%, \mathrm{n}=24)$. Over half of the patients $(68.4 \%$, $\mathrm{n}=26)$ had a smoking history. Twenty patients $(52.6 \%)$ had lymph node metastasis at diagnosis. The TNM stage for all patients was listed as stage I $(36.8 \%, \mathrm{n}=14)$, II $(31.6 \%, \mathrm{n}=12)$, and III $(31.6 \%, \mathrm{n}=12)$. The main pathological types were adenocarcinoma (ADC, $50 \%, \mathrm{n}=19)$, $\mathrm{SCC}(34.2 \%, \mathrm{n}=13)$,
Table 2 Patient characteristics

\begin{tabular}{|c|c|c|}
\hline Variables & $\mathbf{n}$ & $\%$ \\
\hline \multicolumn{3}{|l|}{ Gender } \\
\hline Male & 26 & 68.4 \\
\hline Female & 12 & 31.6 \\
\hline \multicolumn{3}{|l|}{ Age (years) } \\
\hline$<60$ & 24 & 63.2 \\
\hline$\geq 60$ & 14 & 36.8 \\
\hline \multicolumn{3}{|l|}{ Smoking } \\
\hline Never & 12 & 31.6 \\
\hline Ever & 26 & 68.4 \\
\hline \multicolumn{3}{|l|}{ T stage } \\
\hline TI or 2 & 32 & 84.2 \\
\hline T3 or 4 & 6 & 15.8 \\
\hline \multicolumn{3}{|l|}{$\mathrm{N}$ stage } \\
\hline No & 18 & 47.4 \\
\hline $\mathrm{NI}$ or 2 & 20 & 52.6 \\
\hline \multicolumn{3}{|l|}{ TNM } \\
\hline I & 14 & 36.8 \\
\hline II or III & 24 & 63.2 \\
\hline \multicolumn{3}{|l|}{ Pathology } \\
\hline ADC & 19 & 50.0 \\
\hline SCC & 13 & 34.2 \\
\hline Others & 6 & 15.8 \\
\hline \multicolumn{3}{|c|}{ Chemotherapy } \\
\hline Yes & 16 & 42.1 \\
\hline No & 22 & 57.9 \\
\hline \multicolumn{3}{|c|}{ Radiotherapy } \\
\hline Yes & 3 & 7.9 \\
\hline No & 35 & 92.1 \\
\hline
\end{tabular}

Abbreviations: ADC, adenocarcinoma; SCC, squamous cell carcinoma.

and others $(15.8 \%, \mathrm{n}=6)$. There were $16(42.1 \%)$ and $3(7.9 \%)$ patients receiving the treatment of postoperative adjuvant chemotherapy and radiotherapy, respectively.

\section{CTAs expression}

The optimal cut-off value for 5 CTAs based on ROC analysis result was as follows: 2,391.75 for MAGEA1 (AUC $=0.739$; 95\% CI: $0.580-0.897 ; P=0.012), 27.64$ for MAGEA10 (AUC $=0.667 ; 95 \%$ CI: $0.492-0.841 ; P=0.079$ ), 16.11 for MAGEB2 (AUC $=0.694 ; 95 \%$ CI: 0.524-0.895; $P=0.041$ ), 6,199.82 for $K K-L C-1$ (AUC $=0.667$; $95 \%$ CI: $0.492-0.841$; $P=0.079$ ), and 178.85 for $C T A G 1 A / B$ (AUC $=0.594 ; 95 \%$ CI: $0.409-0.780 ; P=0.320$ ), respectively. High expression of MAGEA1, MAGEA10, MAGEB2, KK-LC-1, and CTAG1A/B was found in $16(42.1 \%), 30$ (79.0\%), 29 (76.3\%), $14(36.8 \%)$, and $26(68.4 \%)$ cases, respectively.

\section{The relationships between CTAs expression and patient characteristics}

Clinicopathological features, including gender, age, smoking history, pathological $\mathrm{T}$ stage, pathological $\mathrm{N}$ stage, TNM 
stage, and pathological types were chosen to compare their relationship with CTAs expression. High expression of $M A G E A 1$ (N0 vs N1-2, $16.7 \%$ vs $65.0 \%, P=0.004$ ), $M A G E A 10$ (N0 vs N1-2, 61.1\% vs $95.0 \%, P=0.016$ ), $M A G E B 2$ (N0 vs N1-2, 55.6\% vs 95.0\%, $P=0.007$ ), and $K K-L C-1$ (N0 vs N1-2, $16.7 \%$ vs $55.0 \%, P=0.020$ ) was closely correlated with lymph node metastasis at diagnosis. Although the difference was not statistically significant, $C T A G 1 A / B$ expression also had a similar trend (N0 vs N1-2, $55.6 \%$ vs $80.0 \%, P=0.164)$. High CTAs expression was seen in patients with pathological N1-2 stage. Additionally, high $M A G E A 10$ (I vs II + III, $57.1 \%$ vs $91.7 \%, P=0.034$ ) and $K K$ $L C$ - 1 (I vs II + III, $14.3 \%$ vs $50.0 \%, P=0.039$ ) expression were associated with advanced TNM stage (Table 3 ). Although no statistical significance was found in the rest 3 CTAs, they all had a similar trend that CTAs had a higher expression rate in patients with advanced stage. In addition, patients with age $\geq 60$ years had a higher $C T A G 1 A / B$ expression rate compared with patients with age $<60$ years $(54.2 \%$ vs $92.9 \%$, $P=0.027)$. There was no significant difference found between CTAs expression and gender, smoking history, pathological T stage, and pathological types.

\section{CTAs expression and OS}

The prognostic roles of all variables in Table 2 and 5 CTAs expression were tested to evaluate their potential prognostic values. The 3 years survival rate for patients with high and low $M A G E B 2$ expression was $58.2 \%$ and $100.0 \%(P=0.033)$, respectively. The 3 years survival rate for patients with high and low $C T A G 1 A / B$ expression was $53.6 \%$ and $100.0 \%$ $(P=0.009)$, respectively. High expression of $M A G E B 2$ and $C T A G 1 A / B$ were correlated with unfavorable prognosis (Figure 1). The 3 years survival rates of patients with high $M A G E A 1, M A G E A 10$, and $K K-L C-1$ expressions were $59.2 \%, 63.3 \%$, and $68.8 \%$, compared with $75.6 \%, 87.5 \%$, and $68.9 \%$ in patients with low corresponding CTAs expression. The expression of $M A G E A 1, M A G E A 10$, and $K K-L C-1$ did not have significant association with the prognosis $(P>0.05)$. In addition, $\mathrm{N}$ stage $(P=0.023)$ and TNM stage $(P=0.026)$ were also closely correlated with OS on univariate analysis. No significant survival difference was found in age, gender, smoking history, T stage, pathological types, chemotherapy, and radiotherapy. No variables held statistical significance on multivariate analysis $(P>0.05)$. Our results showed that $M A G E B 2$ and $C T A G 1 A / B$ expression was associated with the prognosis of resected lung cancer patients, but they were not independent prognostic factors.

\section{Subgroup analysis of CTAs expression and OS}

Given CTAs showed a significant higher expression in patients with stage II and III, subgroup analysis based on TNM stage was performed to assess the potential prognostic roles of CTAs expression on selected patients. The variables evaluated were just the same with the analysis for all cases. When the analysis was limited to patients with stage II and III, only high expression of $C T A G 1 A / B$ also had a negative impact on survival (Figure 2, $P=0.031$ ).

Subgroup analysis based on variable pathological types showed that only pathological $\mathrm{N}$ stage $(P=0.019)$ and TNM stage $(P=0.038)$ were closely associated with the prognosis of ADC patients, while no clinicopathological characteristics were associated with the prognosis of non-ADC patients $(P>0.05)$. Although no statistical significance was found between CTAs expression and OS of lung ADC and non-ADC patients, high $C T A G 1 A / B$ expression also had a similar prognostic trend in $\mathrm{ADC}(P=0.052)$ and non-ADC cases $(P=0.100)$.

\section{Validation of CTAs expression in TCGA database}

A total of 962 cases (479 ADC and 483 SCC) in TCGA database were enrolled to validate the aforementioned results in our study. The potential prognostic parameters included pathological types (ADC vs SCC), age ( $<60$ vs $\geq 60$ years), gender (male vs female), tumor stage (I + II vs III + IV), T stage (T1-2 vs T3-4), N stage (N0 vs N1-3) and CTAs expression. For total cases, only tumor stage $(P<0.001)$ $\mathrm{T}(P<0.001)$ and $\mathrm{N}(P<0.001)$ were closely associated with OS on univariate analysis, while no CTAs expression was correlated with OS $(P>0.05)$. When all parameters were evaluated in multivariate analysis, only $\mathrm{T}$ stage (hazard ratio $[\mathrm{HR}]=1.525,95 \% \mathrm{CI}: 1.131-2.057, P=0.006)$ and $\mathrm{N}$ stage $(\mathrm{HR}=1.438,95 \% \mathrm{CI}: 1.131-1.828, P=0.003)$ held statistical significance.

Subgroup analysis was also performed based on variable pathological types. In ADC patients, tumor stage $(P<0.001)$, T stage $(P<0.001), \mathrm{N}$ stage $(P<0.001)$, and $C T A G 1 B$ $(P=0.047)$ were closely associated with the prognosis of patients on univariate analysis (Figure 3). When all parameters were evaluated in multivariate analysis, MAGEA1 $(\mathrm{HR}=1.487$, 95\% CI: 1.006-2.197, $P=0.046), C T A G 1 B(\mathrm{HR}=2.733,95 \%$ CI: $1.348-5.541, P=0.005)$, T stage $(\mathrm{HR}=1.820,95 \% \mathrm{CI}$ : $1.170-2.829, P=0.008)$, and $\mathrm{N}$ stage $(\mathrm{HR}=2.246,95 \% \mathrm{CI}$ : 1.564-3.224, $P<0.001$ ) were all found to be independent prognostic factors of lung ADC patients. In SCC patients, 


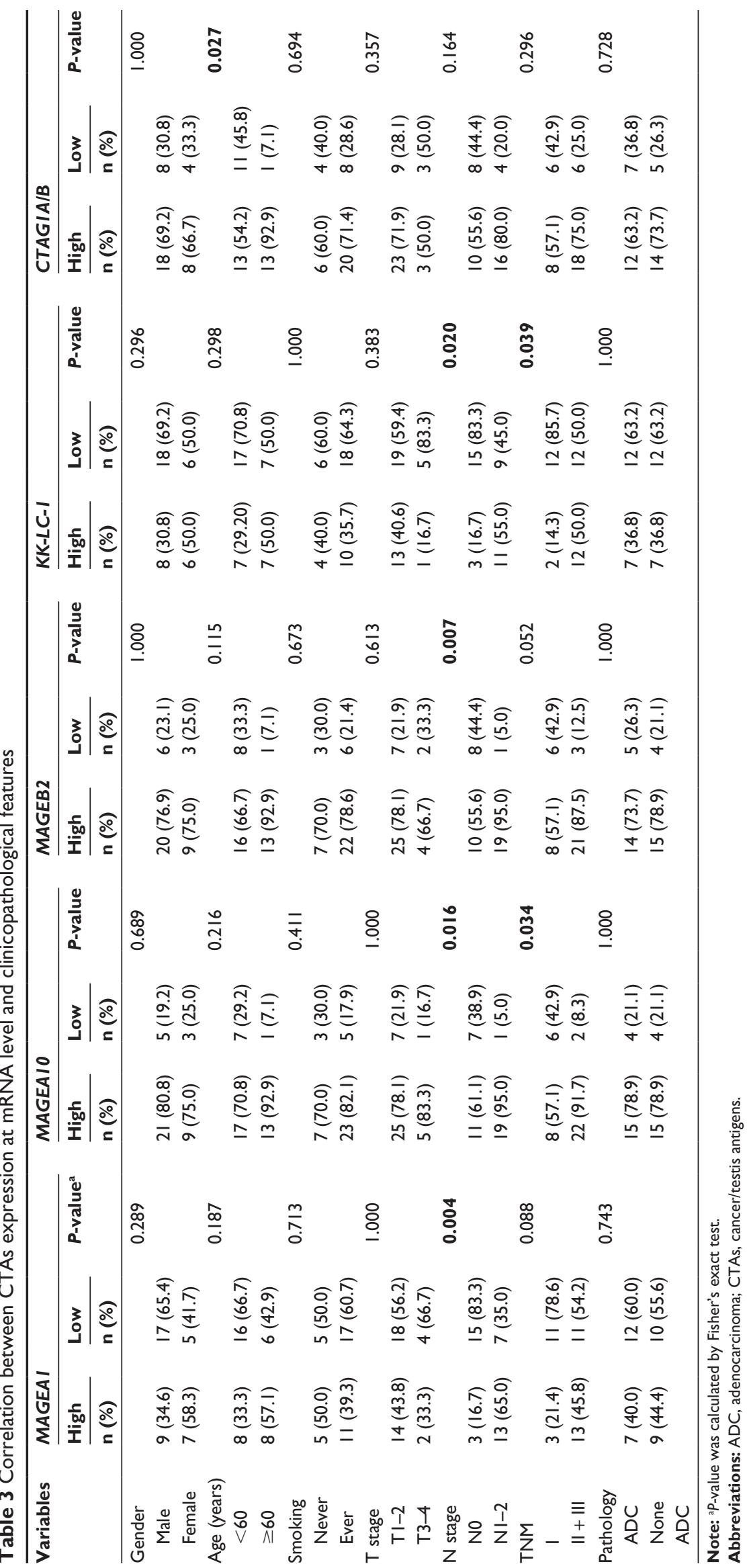



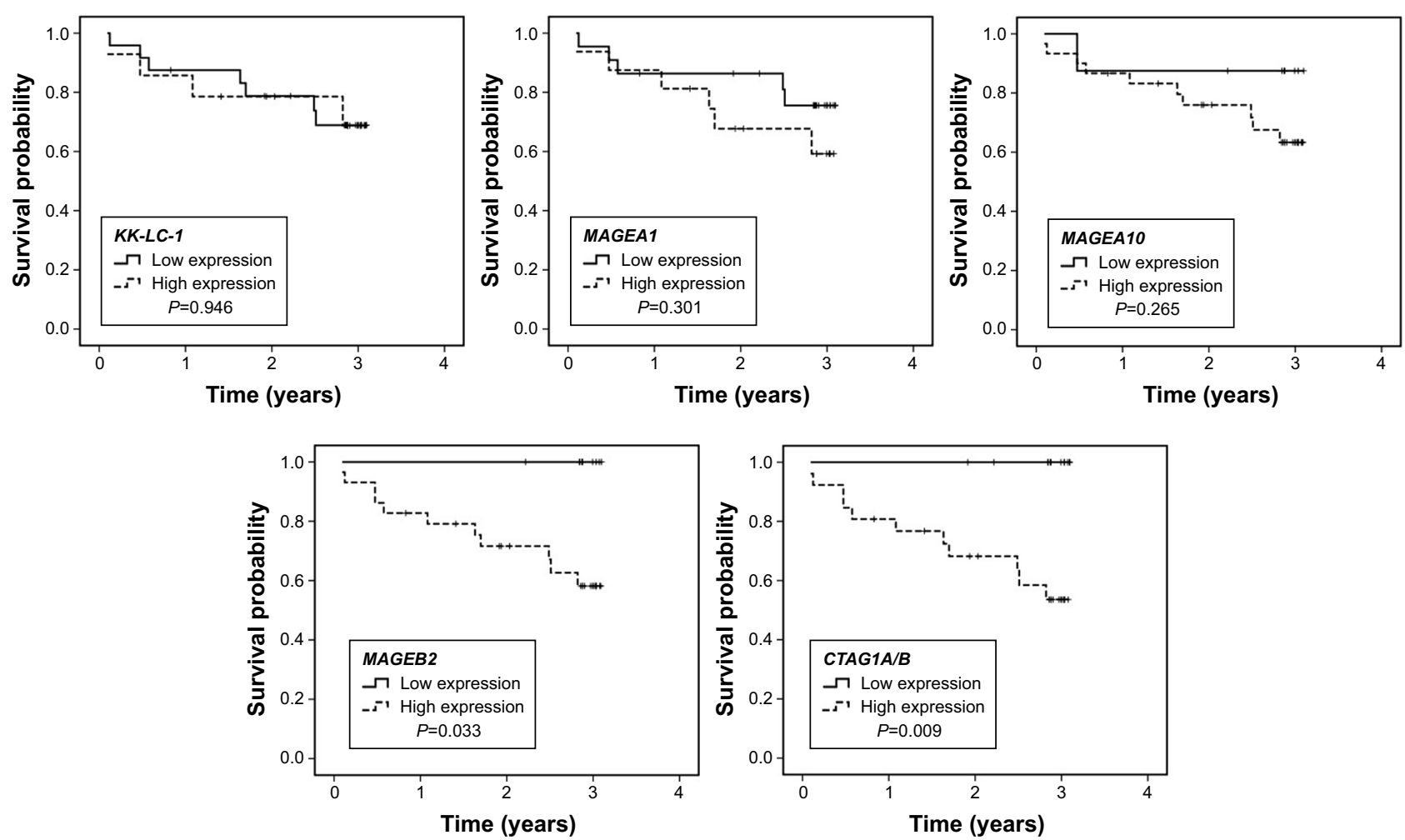

Figure I Overall survival curves of all patients according to CTAs expression.

Note: High MAGEB2 and CTAGIA/B expression showed poorer prognosis than low expression $(P<0.05)$, while the expression of the other 3 CTAs showed no significant correlations with survival $(P>0.05)$.

Abbreviation: CTAs, cancer/testis antigens.
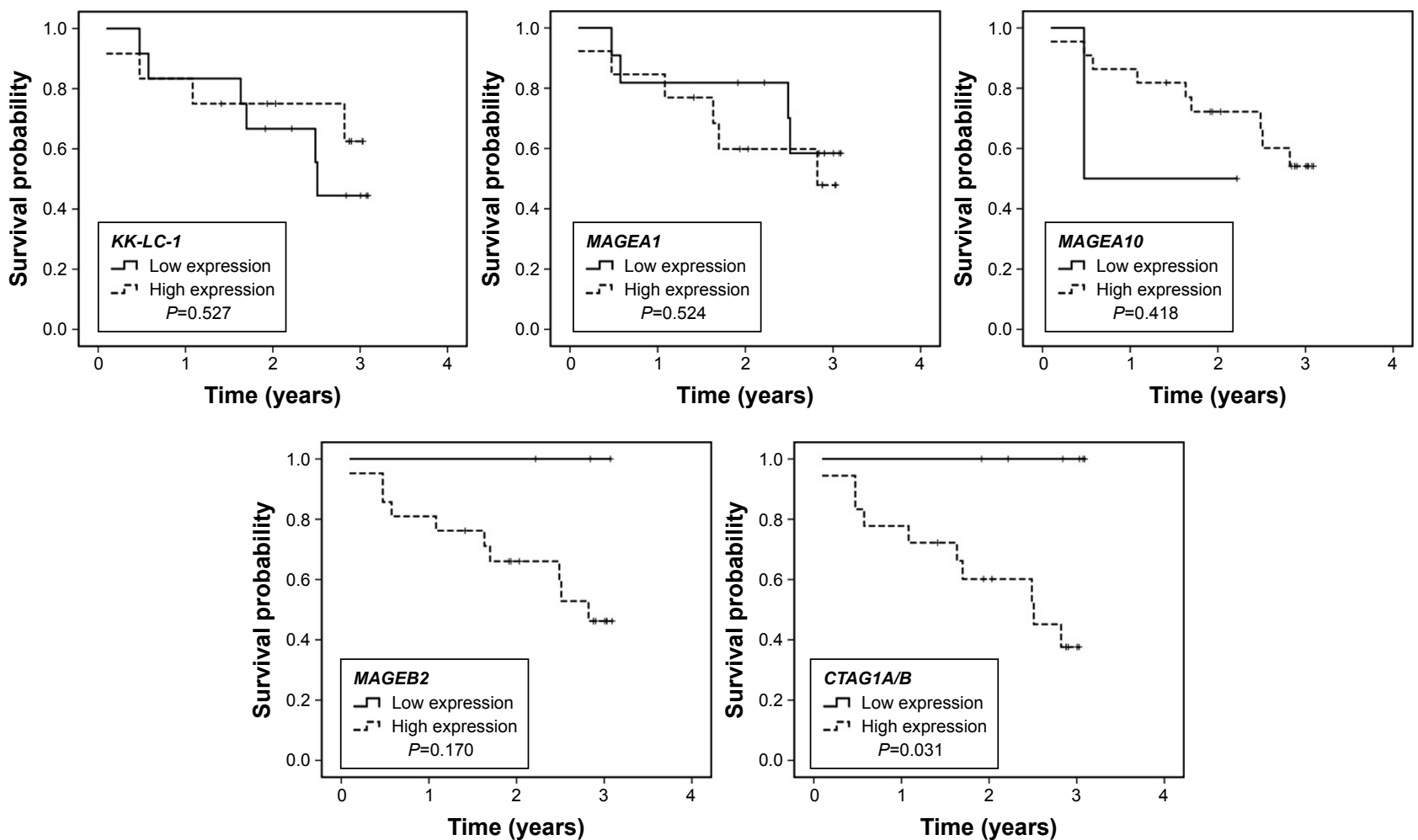

Figure 2 Overall survival curve of patients with pathological stage II and III according to CTAs expression.

Note: In patients with stage II and III, high expression of CTAGIA/B showed poorer prognosis than low expression $(P=0.03 \mathrm{I})$.

Abbreviation: CTAs, cancer/testis antigens. 


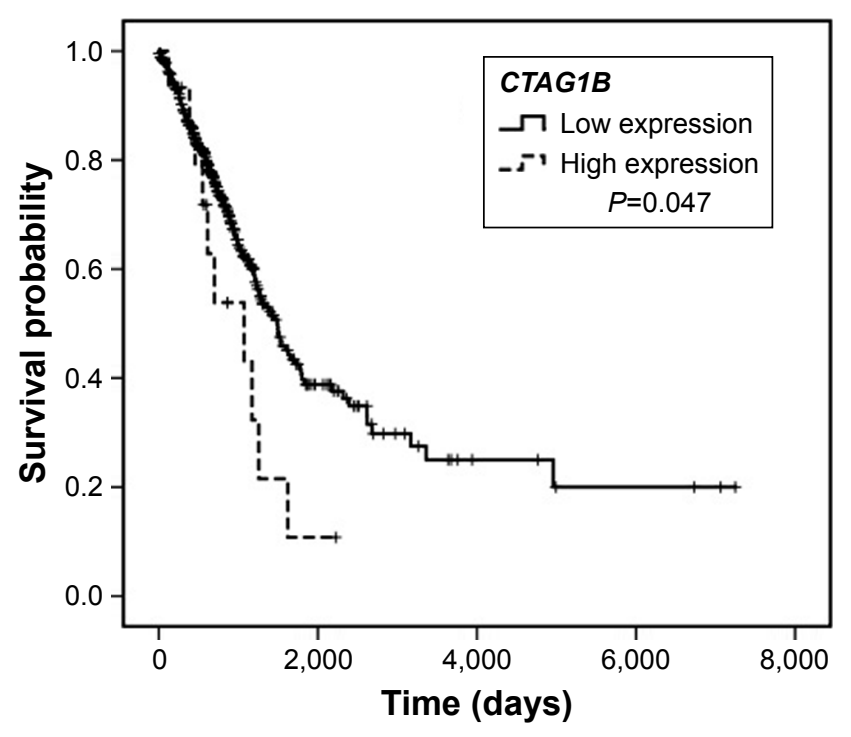

Figure 3 Negative prognostic role of CTAGIB in lung adenocarcinoma demonstrated by The Cancer Genome Atlas database.

only tumor stage $(P=0.012)$ and $\mathrm{T}$ stage $(P=0.004)$ were closely associated with the prognosis of patients, and no parameters were found to have statistical significance on multivariate analysis.

\section{Discussion}

In our study, we found that high expression rate of these 5 CTAs ranged $36.8 \%-79.0 \%$. CTAs expression at mRNA level was closely associated with pathological $\mathrm{N}$ and TNM stages. Patients with stage II-III and lymph node metastasis had a higher CTAs expression rate compared with patients with stage I and no lymph node metastasis. In addition, $C T A G 1 A / B$ expression had a negative impact on OS of resected lung cancer patients. TCGA database analysis showed that the negative prognostic role of $C T A G 1 A / B$ was mainly induced by $C T A G 1 B$, and high $C T A G 1 B$ expression was an independent prognostic factor of lung ADC cases.

More and more attention has been paid to the study of immunotherapy and targeted therapy due to the limited benefits of conventional chemotherapy and the associated toxicities. $^{2}$ CTAs represent potential candidates for the management of cancers due to their restricted expression in cancer and immune-privileged tissues, such as testis and placenta. These immune-privileged tissues cannot present CTAs because of the lacking expression of HLA class I molecules, and this leads to the escape of effector T cell attack. Previous studies have demonstrated the anti-tumor effect of immunotherapy. ${ }^{10-12,25}$ Immunotherapy has 2 significant benefits. On the one hand, if immunotherapy shows great advantages over conventional therapies, patients can benefit more from treatment with immunotherapy. On the other hand, if immunotherapy has as much effect as conventional therapies, that is also good for patients because the toxicities of immunotherapy are usually less. Both the conditions have significant effect on cancer patients.

One notable characteristic of CTAs is their heterogeneity in variable malignancies. Lung cancer belongs to CTA-rich cancers due to its high frequency of CTAs expression. The frequency of 5 tested CTAs in our study varies largely, ranging 36.8\%-79.0\%. Regional and racial difference may influence CTAs expression. The $K K-L C-1$ (36.8\% vs 33.0\%) and MAGEA1 (42.1\% vs 31.0\%) expression is similar with previous studies, while the MAGEA10 (79.0\% vs $28.0 \%)$ expression varies largely with previous studies. ${ }^{22,26}$ In our study, $M A G E B 2$ and $C T A G 1 A / B$ expression frequency represents $76.3 \%$ and $68.4 \%$, which is higher than that in TCGA database. The majority of patients involved in TCGA database were White $(n=722)$, Black and African American $(n=79)$, American Indian $(n=1)$, and unknown origins $(\mathrm{n}=145)$, while only 15 cases were of Asian origin. The TCGA database shows that CTAG1A (13.3\% vs $11.0 \%)$ and $C T A G 1 B$ (6.7\% vs 4.4\%) expression in Asians was higher than non-Asian race. All patients enrolled in our study were of Asian origin, and CTAs expression heterogeneity was significant in variable race.

In our study, CTAs expression was closely associated with pathological lymph node metastasis and advanced tumor stage. Patients with stage II and III show a higher CTAs expression compared with patients with stage I, and this is similar with previous studies. ${ }^{22,27} \mathrm{We}$ also find that patients with lymph node metastasis have a higher CTAs expression frequency compared with patients without lymph node metastasis. A previous study demonstrates that $M A G E C 1$ and $M A G E C 2$ expression can independently predict lymph node metastasis. ${ }^{28}$ More lymph node metastases are found in patients with positive expression of $M A G E C 1$ and $M A G E C 2$, which is in favor of our results.

Cancer stem cells (CSCs) play an important role in cancer invasion and metastasis. ${ }^{29}$ Previous studies have demonstrated that several CTAs involving MAGEB2 and NY-ESO-1 $(C T A G 1 B)$ show preferential expression in CSCs compared with non-CSCs. ${ }^{30}$ They can be regarded as cancer/testis/ stem genes, which are closely associated with the progress of tumor migration, invasion, and the formation of secondary metastatic lesions. ${ }^{14,31,32}$ In our study, the results show that $M A G E B 2$ and $C T A G 1 A / B$ are closely correlated with the prognosis of resected lung cancer patients. In subgroup analysis, $C T A G 1 A / B$ expression still shows unfavorable 
prognosis in patients with stage II and III. This can be partly interpreted by the theory mentioned previously. The TCGA database analysis shows that the negative prognostic role of $C T A G 1 A / B$ is mainly induced by $C T A G 1 B$, and high $C T A G 1 B$ expression is an independent negative prognostic factor of lung ADC patients. Unfortunately, the role of $M A G E B 2$ is not confirmed by TCGA database analysis.

Our study aims at exploring CTAs expression frequency at mRNA level in resected lung cancer and analyzes their relationship with clinicopathological characteristics. There are some limitations of our study. First, the surgical samples in our study are only 38 and the results need to be demonstrated in larger samples. Second, we do not assess the CTAs expression at protein level due to the lack of enough samples. In addition, there may be some limitations present due to the retrospective features of our study.

\section{Conclusion}

In summary, high $M A G E A 1, M A G E A 10, M A G E B 2, K K-$ $L C$ - 1 , and $C T A G 1 A / B$ expression at mRNA level range is $36.8 \%-79.0 \%$. CTAs represent potential candidates for cancer immunotherapy and their expression is closely associated with pathological lymph node metastasis and tumor stage. High $C T A G 1 A / B$ expression is a negative prognostic factor in resected lung cancer patients, and its negative role is mainly induced by $C T A G 1 B$ based on TCGA database analysis. High $C T A G 1 B$ expression is an independent negative prognostic factor of lung ADC patients.

\section{Acknowledgments}

We thank Ke Pan and Cassian Yee (Departments of Melanoma Medical Oncology and Immunology, MD Anderson Cancer Center, Houston, Texas) for providing direction to this project.

This work was supported by the National Natural Scientific Foundation of China (numbers 81673007, 81572824 and 81773133) and the Postdoctoral Scientific Research Developmental Fund of Heilongjiang Province (LBH-Q16155).

\section{Disclosure}

The authors report no conflicts of interest in this work.

\section{References}

1. Torre LA, Bray F, Siegel RL, Ferlay J, Lortet-Tieulent J, Jemal A. Global cancer statistics, 2012. CA Cancer J Clin. 2015;65(2):87-108.

2. Zhong WZ, Wang Q, Mao WM, et al. Gefitinib versus vinorelbine plus cisplatin as adjuvant treatment for stage II-IIIA (N1-N2) EGFR-mutant NSCLC (ADJUVANT/CTONG1104): a randomised, open-label, phase 3 study. Lancet Oncol. 2018;19(1):139-148.
3. Borghaei H, Paz-Ares L, Horn L, et al. Nivolumab versus Docetaxel in Advanced Nonsquamous Non-Small-Cell Lung Cancer. N Engl J Med. 2015;373(17):1627-1639.

4. Brahmer J, Reckamp KL, Baas P, et al. Nivolumab versus Docetaxel in Advanced Squamous-Cell Non-Small-Cell Lung Cancer. $N$ Engl $J$ Med. 2015;373(2):123-135.

5. Horn L, Spigel DR, Vokes EE. Nivolumab Versus Docetaxel in Previously Treated Patients With Advanced Non-Small-Cell Lung Cancer: Two-Year Outcomes From Two Randomized, Open-Label, Phase III Trials (CheckMate 017and CheckMate 057). J Clin Oncol. 2017;35(35): 3924-3933.

6. Siegel R, Ma J, Zou Z, Jemal A. Cancer statistics, 2014. CA Cancer J Clin. 2014;64(1):9-29.

7. Nakagawa K, Noguchi Y, Uenaka A, et al. XAGE-1 expression in nonsmall cell lung cancer and antibody response in patients. Clin Cancer Res. 2005;11(15):5496-5503.

8. Curigliano G, Viale G, Ghioni M, et al. Cancer-testis antigen expression in triple-negative breast cancer. Ann Oncol. 2011;22(1):98-103.

9. Kim J, Reber HA, Hines OJ, et al. The clinical significance of MAGEA3 expression in pancreatic cancer. Int J Cancer. 2006;118(9): 2269-2275.

10. Yee C, Thompson JA, Byrd D, et al. Adoptive T cell therapy using antigenspecific CD8+ T cell clones for the treatment of patients with metastatic melanoma: in vivo persistence, migration, and antitumor effect of transferred T cells. Proc Natl Acad Sci U S A. 2002;99(25):16168-16173.

11. Chapuis AG, Thompson JA, Margolin KA, et al. Transferred melanomaspecific $\mathrm{CD} 8+\mathrm{T}$ cells persist, mediate tumor regression, and acquire central memory phenotype. Proc Natl Acad Sci U S A. 2012;109(12): 4592-4597.

12. Chapuis AG, Ragnarsson GB, Nguyen HN, et al. Transferred WT1reactive $\mathrm{CD} 8+\mathrm{T}$ cells can mediate antileukemic activity and persist in post-transplant patients. Sci Transl Med. 2013;5(174):174ra27.

13. Yang B, O'Herrin SM, Wu J, et al. MAGE-A, mMage-b, and MAGE-C proteins form complexes with KAP1 and suppress p53-dependent apoptosis in MAGE-positive cell lines. Cancer Res. 2007;67(20): 9954-9962.

14. Whitehurst AW. Cause and consequence of cancer/testis antigen activation in cancer. Annu Rev Pharmacol Toxicol. 2014;54:251-272.

15. Barrow C, Browning J, Macgregor D, et al. Tumor antigen expression in melanoma varies according to antigen and stage. Clin Cancer Res. 2006;12(3 Pt 1):764-771.

16. Velazquez EF, Jungbluth AA, Yancovitz M, et al. Expression of the cancer/testis antigen NY-ESO-1 in primary and metastatic malignant melanoma (MM)-correlation with prognostic factors. Cancer Immun. 2007;7:11.

17. Fukuyama T, Hanagiri T, Takenoyama M, et al. Identification of a new cancer/germline gene, KK-LC-1, encoding an antigen recognized by autologous CTL induced on human lung adenocarcinoma. Cancer Res. 2006;66(9):4922-4928.

18. Ohue Y, Kurose K, Mizote Y, et al. Prolongation of overall survival in advanced lung adenocarcinoma patients with the XAGE1 (GAGED2a) antibody. Clin Cancer Res. 2014;20(19):5052-5063.

19. Ayyoub M, Scarlata CM, Hamaï A, Pignon P, Valmori D. Expression of MAGE-A3/6 in primary breast cancer is associated with hormone receptor negative status, high histologic grade, and poor survival. J Immunother. 2014;37(2):73-76.

20. Gure AO, Chua R, Williamson B, et al. Cancer-testis genes are coordinately expressed and are markers of poor outcome in non-small cell lung cancer. Clin Cancer Res. 2005;11(22):8055-8062.

21. Laban S, Atanackovic D, Luetkens T, et al. Simultaneous cytoplasmic and nuclear protein expression of melanoma antigen-A family and NY-ESO-1 cancer-testis antigens represents an independent marker for poor survival in head and neck cancer. Int J Cancer. 2014;135(5): $1142-1152$.

22. Shigematsu Y, Hanagiri T, Shiota H, et al. Clinical significance of cancer/testis antigens expression in patients with non-small cell lung cancer. Lung Cancer. 2010;68(1):105-110. 
23. Scanlan MJ, Simpson AJ, Old LJ. The cancer/testis genes: review, standardization, and commentary. Cancer Immun. 2004;4:1.

24. Detterbeck FC, Boffa DJ, Kim AW, Tanoue LT. The Eighth Edition Lung Cancer Stage Classification. Chest. 2017;151(1):193-203.

25. Atanackovic D, Arfsten J, Cao Y, et al. Cancer-testis antigens are commonly expressed in multiple myeloma and induce systemic immunity following allogeneic stem cell transplantation. Blood. 2007;109(3): 1103-1112.

26. Tajima K, Obata Y, Tamaki H, et al. Expression of cancer/testis (CT) antigens in lung cancer. Lung Cancer. 2003;42(1):23-33.

27. Salmaninejad A, Zamani MR, Pourvahedi M, Golchehre Z, Hosseini Bereshneh A, Rezaei N. Cancer/Testis Antigens: Expression, Regulation, Tumor Invasion, and Use in Immunotherapy of Cancers. Immunol Invest. 2016;45(7):619-640.

28. Curioni-Fontecedro A, Nuber N, Mihic-Probst D, et al. Expression of MAGE-C1/CT7 and MAGE-C2/CT10 predicts lymph node metastasis in melanoma patients. PLoS One. 2011;6(6):e21418.
29. Fazilaty H, Gardaneh M, Bahrami T, Salmaninejad A, Behnam B. Crosstalk between breast cancer stem cells and metastatic niche: emerging molecular metastasis pathway? Tumour Biol. 2013;34(4):2019-2030.

30. Cronwright G, Le Blanc K, Götherström C, Darcy P, Ehnman M, Brodin B. Cancer/testis antigen expression in human mesenchymal stem cells: down-regulation of SSX impairs cell migration and matrix metalloproteinase 2 expression. Cancer Res. 2005;65(6):2207-2215.

31. Ghafouri-Fard S. Expression of cancer-testis antigens in stem cells is it a potential drawback or an advantage in cancer immunotherapy. Asian Pac J Cancer Prev. 2015;16(7):3079-3081.

32. Yamada R, Takahashi A, Torigoe T, et al. Preferential expression of cancer/testis genes in cancer stem-like cells: proposal of a novel sub-category, cancer/testis/stem gene. Tissue Antigens. 2013;81(6): $428-434$.

\section{Publish your work in this journal}

OncoTargets and Therapy is an international, peer-reviewed, open access journal focusing on the pathological basis of all cancers, potential targets for therapy and treatment protocols employed to improve the management of cancer patients. The journal also focuses on the impact of management programs and new therapeutic agents and protocols on

\section{Dovepress}

patient perspectives such as quality of life, adherence and satisfaction. The manuscript management system is completely online and includes a very quick and fair peer-review system, which is all easy to use. Visit http://www.dovepress.com/testimonials.php to read real quotes from published authors. 